\title{
Study on Computer-aided Optimized Design of Structured Cabling Construction Drawing of Intelligent Building
}

\author{
Qiu Lin \\ Nanchang Key Laboratory of Material and Structure Detection, Jiangxi University of Technology
}

Keywords: Structured Cabling; Optimization Design; AutoCAD; AutoCAD VBA

\begin{abstract}
With the vigorous development of intelligent building, the structured cabling system is more and more widely applied. At present, in the process of structured cabling systems engineering design, the construction drawing is basically designed by using the common drawing software such as AutoCAD, in which the high-speed computing power of computer is not fully applied, the manual intervention is high, and efficiency is low. In order to improve the quality and efficiency of the structured cabling system design, and promote the application of CAD technology and optimization technology in intelligent building structured cabling system design, the paper introduced the development situation of structured cabling technology and the application of CAD technology in structured cabling industry, elaborated the each cabling subsystem design of structured cabling system construction drawing, and illustrated the situation of application for optimization method in the CAD construction drawing of structured cabling system. The method of AuotCAD secondary development, the thoughts and methods of applying optimization method in the structured cabling system design are used in the study, which opened up a new research direction for the later study on $\mathrm{CAD}$ of structured cabling system.
\end{abstract}

\section{Introduction}

With the advent of information age and vigorous development of intelligent building, the application of structured cabling system is more and more wide. Under the good opportunity of the advent of modern computer technology, optimization computing method and modern graphics technology, the research topic was put forward in order to adapt to the rapid development of construction technology of structured cabling system, improve the quality and efficiency of structured cabling system design, promote the application of CAD technology and optimization technology in the intelligent building structured cabling system construction.

Structured cabling system is the important infrastructure of the intelligent building and building group. Structured Cabling System is also known as Generic Cabling System (GCS). It is a set of open cabling system, and the "nervous system" of intelligent building. In theory, it can support almost all of the data, voice equipment and various kinds of communication protocol. Structured cabling system is superior to the traditional cabling system in many ways such as its compatibility, openness, flexibility, reliability, advancement and economy. What's more, in the aspect of design, construction and maintenance, it brings a lot of convenience to users.

The development of structured cabling system is closely related to the building automation in the first place. In the early 1950s, some developed countries adopt electronic device control system in the large high-rise buildings. Since the 1980s, with the continuous development of science and 
technology, especially the integration and development of communications, computer network, control and graphics display technology, increase of high-rise building service function and objective demand, expanding information content and type in the building, the information transmission system of the building has become bigger and bigger, and the traditional cabling system has been unable to meet the need.

It has been about 10 years since the wide application of structured cabling technology in the intelligent building, and the study and application of structured cabling system CAD is still in its infancy. Although the development of structured cabling system CAD software is under way by the foreign scientific research institutions and system integrators, some of the developed CAD software for structured cabling system is for the project design; the CAD software for construction drawing is very few, and there is no one of them being widely used.

At present, the intelligent building is in the climax of construction around the world. It is estimated that more than half of the intelligent build ings will be built in China. As the "central nervous" system of intelligent buildings, structured cabling system is the necessary infrastructure of intelligent buildings. More than $70 \%$ of the root cause of intelligent system paralysis is the bad cabling. Therefore, it is necessary to strengthen the study on the design of structured cabling system and construction methods.

The paper adopted AutoCADZO02 as graphics platform, made use of optimization method and AutoCAD secondary development technology, and studied the structured cabling construction drawing CAD design and optimization of intelligent building. The basic thought and main work content in the paper include:

(1) Study and use "the shortest path problem" principle and calculation method of network optimization technology in the optimization method while consider the feasibility of the project, and make optimal calculation selection of structured cabling system route. Select the optimal cabling route of possible cabling route in the horizontal cabling subsystem based on calculation.

(2) Study and use the selection principle of optimal positioning, and make optimal positioning option design of the most important part (such as computer room, telephone mainframe room, equipment room or BD, transfer room or FD and weak current shaft) in the structured cabling design.

(3) Study and use the Visual LISP development technology to define new AutoCAD commands; improve the design efficiency of engineering and technical personnel, shorten the design cycle, and save the engineering and technical personnel from the traditional complicated construction plan and the drawing, design and calculation of system chart.

Design of structured cabling system construction drawing. Structured cabling system in general is divided into six subsystems: workspace subsystem, distribution horizontal subsystem, main line (vertical) subsystem, equipment room subsystem, management subsystem and building group subsystem. The engineering design of intelligent building and building group should select corresponding configuration of structured cabling system according to the actual function need of intelligent building, and consider its long-term development. When network usage requirement of the intelligent building is not clear, in general case, we should design according to the system configuration rank in accordance with the <specification of integrated cabling system engineering design of building and building group>.

Twisted-pair used in the structured cabling system project can be divided into Shielded Twisted Pair and Unshielded Twisted Pair. The external shielding layer exists in the shielded twisted-pair rather than in unshielded twisted pair. The role of shielding layer in shielded twisted pair is to reduce 
the external electromagnetic radiation energy of shielded twisted pair cable, improve the resistance to external electromagnetic interference, and better guarantee the accuracy of data transmission. Unshielded twisted pair has outstanding features such as easy installation, light, thin and flexible; the external diameter of cable is relatively small because of no shield layer; there is certain anti-interference ability by adopting the feature of balanced transmission; support high-speed data transmission; with a wide range of standard support, almost all standards in IEEE802 support UTP media; standard structured cabling system transmission medium; transmission distance less than 100 m; pass EMC testing; relatively cheap price.

Shielded twisted-pair has the following several prominent features: strong mounting technique; both balanced transmission and shielded transmission protection; pass the EMC test and conform to EMC trend; strong anti-interference ability and strong anti-radiation ability from outside; suitable for strong interference environment and more sensitive to electromagnetic interference environment; relatively expensive price; less than $100 \mathrm{~m}$ transmission distance.

Unshielded twisted pair has the essential feature of balanced transmission, and the transmission performance can be guaranteed in the case of better electromagnetic compatibility of EMC. In the case of electromagnetic interference, on the premise of correct grounding of FTP, it can effectively prevent the outside electromagnetic interference to FTP system, and guarantee the normal operation of shielding system under the severely interfered environment. Therefore, according to this principle, in actual engineering, the building is in the campus, general administrative organs or institutional unit out of consideration of higher requirements to future transmission performance, and electromagnetic compatibility of EMC. Generally, there are two alternatives; one is shielding plan and the other is unshielded plan, which is decided according to economic strength, the system performance requirements, and EMC environmental consciousness, etc. On the contrary, if the project is at the airport, radio and television transmitters, power plant, dock or other environment with severe electromagnetic interference, only shielded twisted pair system can be adopted. In addition, considering the relatively high requirement to anti-electromagnetic interference and anti-electromagnetic radiation in the secret service such as military organization, banks, and government agencies, the shielded twist pair system can only be used.

Design of equipment room subsystem. Equipment room is the place that sets up appropriate telecommunication equipment and computer network equipment as well as building cabling devices for network management. For structured cabling engineering design, the main building cabling equipment installed in equipment room consists of cables, connectors and related support hardware, and interconnects all sorts of utility system equipment through cables. All kinds of host devices such as telephone and computer, and entrance facility can be put together. The main devices in equipment room are digital program-controlled switches, computer network equipment, server, building automation equipment, etc. They can be put together, also can be set respectively. In larger structured cabling systems, computer equipment, digital program-controlled switches, building automation equipment hosts are set in the computer room respectively; hardware devices that is closely related with structured cabling are placed in the equipment room; the machine room of computer network equipment is set near the equipment room. The ideal setting is to set the equipment room such as computer room and switching equipment room in the same floor, as it is convenient for management and reduces investment outlay. The location and size of the equipment room should be decided by comprehensive consideration such as the number, scale, and best network center of the equipment. Equipment room should have enough installation space, which includes: program-controlled digital 
subscriber telephone switching system, the computer host, the switching equipment of the whole building, etc. There must be an equipment room inside a building.

The design method of equipment room is as follows:

(1) Determine the position of the equipment room:

Try to set the middle position of best network in the main line of structured cabling system, which is the middle of the building, and it is easy to install the grounding device; keep away from all electromagnetic interference sources, avoid strong vibration source and strong noise source, and keep away from harmful gas source, corrosive, flammable, explosive and so on. For the convenience of loading and transporting equipment of equipment room, don't be too far away from the service elevator, and try not to set it in the basement of the building.

(2) Determine the usable area of equipment room

The equipment room not only stores devices such as PABX, computer host and the intersection unit of the whole building, but also provides convenience for the management and operation of the staffs. There are two ways of usable area design: first, the usable area of equipment room is 5 to 7 times of the total floor area of all the equipment, and the formula is as follows:

$\mathrm{S}=(5 \sim 7) \sum \mathrm{S}_{\mathrm{b}}$

In the design formula of structured cabling system construction drawing, $S$ stands for the usable area of equipment room; $S_{b}$ stands for the area of the equipment installed in the equipment room and be related with structured cabling system; $\sum \mathrm{S}_{\mathrm{b}}$ stands for the sum of floor area of all the equipment in the equipment room.

The second method is to calculate according to the thought of average, namely the multiply of total number of equipment and empirical coefficient of the average equipment floor area, and the formula is:

$\mathrm{S}=\mathrm{KN}$

In the formula, $\mathrm{S}$ stands for the usable area of the equipment room; $\mathrm{K}$ is the coefficient, generally valued as $4.5-5.5 \mathrm{~m}^{2} / \mathrm{set}$; $\mathrm{N}$ stands for the total number of the equipment in the equipment room.

In the above two methods, the minimum usable area of the equipment room should not be less than $20 \mathrm{~m}^{2}$.

(3)Determine the building structure:

The main considerations of building structure include equipment placement, entrance and exit, bearing, etc. The clear height of the equipment room can be taken from $2.55 \mathrm{~m}$ to $3.2 \mathrm{~m}$. The height of the door of equipment room should be greater than $2.1 \mathrm{~m}$, and the width of the door should be greater than $0.9 \mathrm{~m}$. The loading of floor varies from equipment, and the equivalent uniform live load of the floor should be greater than $5 \mathrm{Nk} / \mathrm{m}^{2}$ at large.

Optimization design method of structured cabling system route. Structured cabling system is the information transmission channel composed by cables and related connection hardware, and the digital signal compiled by electromagnetic or electronic code is transmitted in the channel. The performance of information transmission in the structured cabling system depends not only on the cabling construction technology, but also on the cables adopted, quality of related connection 
hardware, selection of cabling route, distance of signal transmission or length of cabling, etc.

In order to ensure the signal transmission performance and quality in structured cabling system, structured cabling system requires "cabling transmission channel certification test" in construction inspection. The test is to test and compare the structured cabling system item by item based on certain standard so as to determine whether the structured cabling system meets the design standards. The transmission parameters that have to be tested in certification tests include wiring diagram, length, damping and near-end cross-talk. "Damping" is the measure of the transmission loss of the signal along the link. With the increase of the length of link, the damping will increase, namely the more signals are lost. When the signal attenuation reaches to a certain degree, it will cause the unreliable information in the link transmission. Therefore, the testing standard specifies the allowable values of the basic link and channel attenuation so as to ensure the reliability of information transmission.

The essence of the optimization problems in engineering is to find the extreme value of objective function under certain conditions, and the constraint conditions are put forward according to various factors such as the technical requirements of different project object, investment cost, the maintenance and management, etc. The whole structured cabling system is large and complex system engineering; in the CAD design of structured cabling system in intelligent building, one of the applications of the optimization technology reflect distinctly in the solution to the optimization of structured cabling system route.

The cable route in structured cabling system should choose the shortest, the safest and the most economical one. The specifications of structured cabling require adopting the open star topology structure and each subsystem is independent mutually. Therefore, structured cabling system adopts modular design method, namely building group cabling subsystem, main line subsystem, horizontal subsystem, the workspace subsystem. In the design of cabling route, optimize respectively and the whole cabling system achieve the best cabling route. According to characteristics of structured cabling system design, optimization design of structured cabling system route should be divided into two parts.

The first part, consider the floor of the building where the computer room, telephone host room, and equipment room are set, consider the design position of the cross connection room in each floor, weak current shaft of structured cabling system in floor plan, namely the selection of optimal position of computer room, equipment room, cross connection room and weak current shaft. This is because at the beginning of the architectural design, before the completion of construction drawing, the designer of structured cabling system should provide the location and size of computer room, telephone host room, equipment room or the floor of DB to the building designers as well as the design location and size of cross connection room, or the FD and weak current shaft of structured cabling system in each floor. Therefore, the route optimization of structured cabling system should determine the optimization layout position of computer room, equipment room, cross connection room and weak current shaft.

The second part, consider the cabling route optimization design of cabling subsystem, main line subsystem, horizontal subsystem, and the workspace subsystem in building group. After the completion of a construction drawing, structured cabling system designer can design the subsystems according to the complete set of construction scheme of buildings and the communication equipment using scheme assumed by users. Because the structured cabling system can adopt the modular design method, the cabling route of building group cabling subsystem, main line subsystem, horizontal 
subsystem, and the workspace subsystem are optimized respectively.

The program implementation on optimization design of structured cabling system route. Route of structured cabling system is a complex system, and the practical optimization design model has the characteristics of hierarchical (multiple) structure, which means the specifications of structured cabling system require adopting open star topology structure, and each subsystem is mutually independent. Therefore, break the structured cabling system into several subsystems, adopt the modular design method, namely optimize respectively the cabling route design of building group cabling subsystem, main line subsystem, horizontal subsystem, and the workspace subsystem, and finally get the best cabling route of the whole cabling system. The modular structure of structured cabling system route optimization design:

The first part, consider the optimization location selection of computer room, equipment room, cross connection room and weak current shaft, namely the floor of computer room, telephone host room and equipment room arranged in the building; consider the optimization design location in floor plan of the cross connection room in each floor, and the weak current shaft in structured cabling system.

The second part, consider the cabling optimization design inside corridor;

The third part, consider the cabling optimization design inside the room.

The modular diagram of the whole optimization design process is shown in the chart 1 .

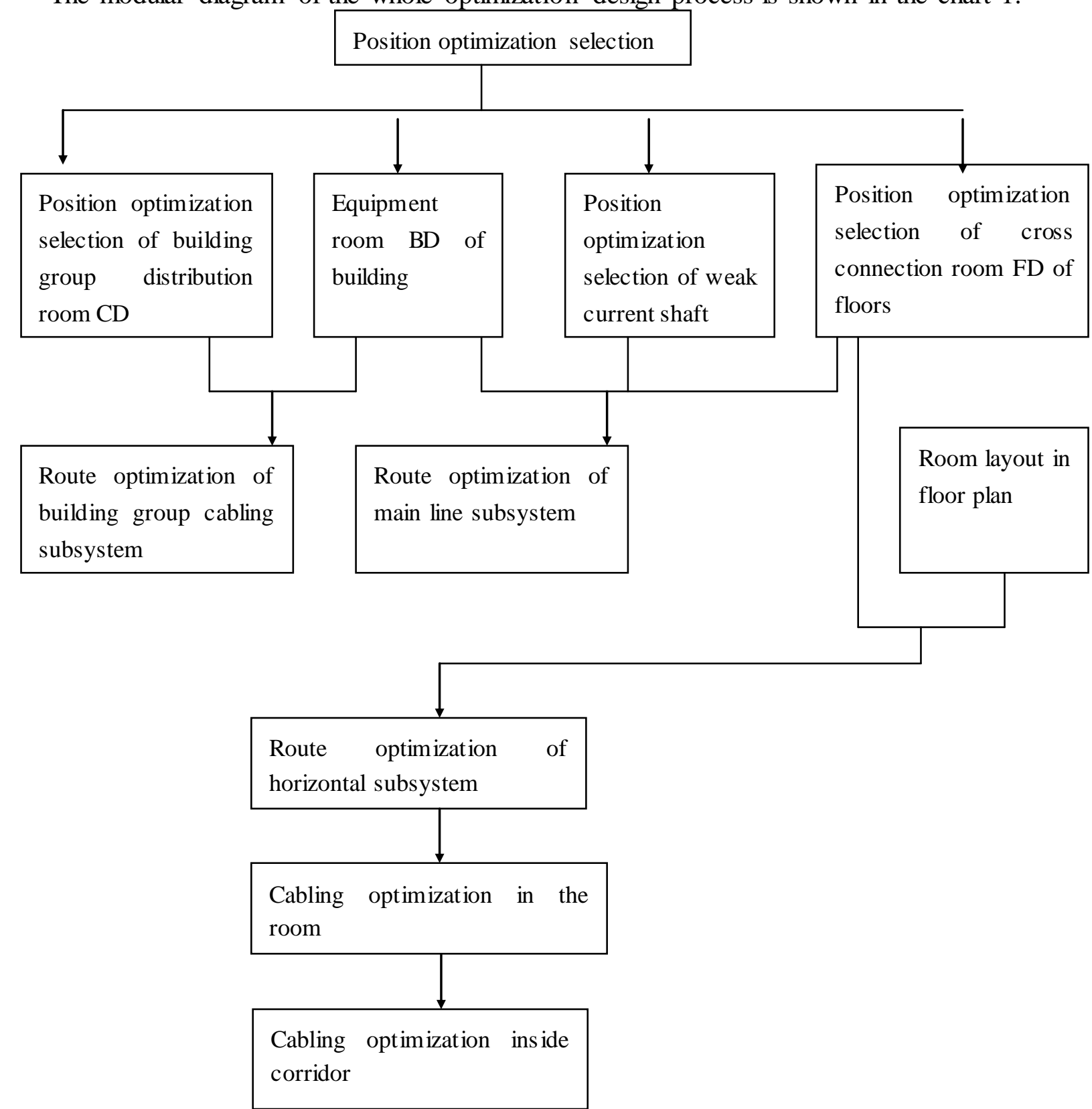

Chart 1 modular diagram of route optimization design in structured cabling system 


\section{Conclusions}

The paper introduced the status of structured cabling technology development, elaborated the design of each cabling subsystem in the structured cabling system construction drawing, and illustrated the situation of application for optimization method in the CAD construction drawing of structured cabling system. What's more, the paper discussed in detail the calculation method of developing optimization method for the cabling route optimization in structured cabling system construction drawing, and expounded the ideas and methods of computer automatic generation in the CAD plan cabling of structured cabling system.

Through in-depth study of this subject, the main achievements are as follows:

(1) Propose to apply the "position optimization selection" principle; in the design of structured cabling system, the optimization selection design in the floor plan of the most important parts are computer room, telephone host room, equipment room or BD floor location as well as the cross connection room or FD in each floor, and weak current shaft of structured cabling system.

(2) In combination with the feasibility of the project implementation, make optimal calculation selection for structured cabling system route. According to the calculation, select the optimal cabling route from floor distribution room to each room in horizontal cabling subsystem, and the optimal cabling route from building group

(3) Define part of the new AutoCAD command by using Visual LISP development technology, which can greatly improve the design efficiency of engineering technicians, and shorten the design cycle.

(4) Based on the optimal calculation, achieve the computer automatic generation of the optimal cabling route from floor distribution room to the entrance point of each room selected in the horizontal cabling subsystem.

(5) Achieve the computer automatic generation of the integrated optimal cabling route from entrance point of room to each information outlet in the plan. Find the features of structured cabling system superior to traditional cabling system.

This paper put forward some thoughts and methods of optimal method applied in structured cabling system, and opened up a new research direction for the later study on CAD design in structured cabling system.

Intelligent building is still in its infancy in China. With the advent of the information era, intelligent buildings will be vigorously developed both at home and abroad. The structured cabling system will be more and more widely used with the development of intelligent building, and its software products will certainly have good application market. Therefore, the continuous development and improvement of the design software in structured cabling system will be accepted by designers, enjoy great popularity in the field of construction, and make contribution to the design and construction of intelligent building in China.

\section{Acknowledgment}

This work was supported by Project on professional and characteristical construction of Jiangxi province 2010 (Civil Engineering) and Project on the planning and construction of disciplines in Jiangxi University of Technology (Structure Engineering) 


\section{References}

[1] Hartsog C H. Computer aided building plan review system and process: U.S. Patent 4,964,060[P]. 1990-10-16.

[2] Koo B, Fischer M. Feasibility study of 4D CAD in commercial construction[J]. Journal of construction engineering and management, 2000, 126(4): 251-260.

[3] Chen Z, Clements-Croome D, Hong J, et al. A multicriteria lifespan energy efficiency approach to intelligent building assessment[J]. Energy and Buildings, 2006, 38(5): 393-409.

[4] Do E Y L. The Right Tool at the Right Time: drawing as an interface to knowledge based design aids[C]//Proceedings of the ACADIA Conference, Design computation: Collaboration, Reasoning, Pedagogy. 1996: 191-199.

[5] Kalay Y E. The impact of information technology on design methods, products and practices[J]. Design studies, 2006, 27(3): 357-380.

[6] Groover M, Zimmers E. CAD/CAM: computer-aided design and manufacturing[M]. Pearson Education, 1983.

[7] Gill P E, Murray W, Saunders M A. SNOPT: An SQP algorithm for large-scale constrained optimization[J]. SIAM journal on optimization, 2002, 12(4): 979-1006.

[8] Ullman D G Dietterich T A. Mechanical design methodology: implications on future developments of computer-aided design and knowledge-based systems[J]. Engineering with Computers, 1987, 2(1): 21-29.

[9] Ozkaya I, Akin Ö. Tool support for computer-aided requirement traceability in architectural design: The case of DesignTrack[J]. Automation in Construction, 2007, 16(5): 674-684.

[10]Alting L, Zhang H. Computer aided process planning: the state-of-the-art survey[J]. The International Journal of Production Research, 1989, 27(4): 553-585. 